\title{
Estrategias para la reducción de las infecciones asociadas a la atención a la salud en el Hospital General Regional \# 251, IMSS, Metepec
}

\author{
Iván Renato Zúñiga-Carrasco ${ }^{1 *}$, María Isabel Estrada-Rodríguez ${ }^{2}$, Jorge Rafael Gamboa-Cardeña ${ }^{3}$ \\ Lilia Cote-Estrada ${ }^{4}$, Juan José de la Fuente-Soto ${ }^{4}$, Beatriz Celia Hernández-Domínguez ${ }^{4}$, \\ Alicia Hernández-Reyes ${ }^{4}$ y Maribel Zepeda-Arias ${ }^{4}$ \\ ${ }^{1}$ Jefe del Servicio de Epidemiología, UMF 223, IMSS, Lerma; '²xdirectora del HGR 251, IMSS, Metepec; ${ }^{3}$ Lider del Proyecto Higiene de Manos, HGR 251, IMSS, \\ Metepec; " ${ }^{4}$ Exlíderes del Modelo Institucional para Prevenir y Reducir las Infecciones Nosocomiales (MIPRN), Dirección Prestaciones Médicas, IMSS
}

\begin{abstract}
RESUMEN
Antecedentes: Las infecciones asociadas a la atención a la salud (IAAS) se asocian con un incremento importante en los costos en el ámbito de la atención hospitalaria y con un aumento importante de la morbilidad. Objetivo: A través de una prueba piloto se tomaron estrategias para reducir las IAAS en el Hospital General Regional 251. Metodología: Se realizó una prueba piloto en el Hospital General Regional 251 que consistió en impulsar el lavado de manos multimodal e implementando estrategias encaminadas a reducir las IAAS. Los resultados de dicha prueba piloto se midieron a través de la información mensual que emite el hospital sobre las IAAS. Se asigno un líder, que coordinó la campaña de higiene de mano a través de la estrategia multimodal implementada por la Organización Mundial de la Salud (OMS). Resultados: Desde 2013 hasta 2017 el número de casos de IAAS fluctuaba entre los 60 y 119 casos, pero al implementar las estrategias disminuyeron las infecciones nosocomiales, siendo una de ellas la correcta clasificación de las mismas. Conclusiones: Las pruebas piloto requieren unos 3-5 años para ver resultados concluyentes, pero con las estrategias implementadas se cambiaron malas prácticas, una de las cuales fue el sobrerregistro de IAAS.
\end{abstract}

Palabras clave: Infección nosocomial. Estrategia. Prueba piloto. Sobrerregistro. Malas prácticas.

\section{ABSTRACT}

Background. Healthcare associated infections (HAI) are an important problem for health systems because of their association with a significant increase in morbidity and costs of hospital care. Objective: To test strategies aimed at reducing HAI in a pilot study at the Regional General Hospital 251 of the Mexican Social Security Institute. Methods: The pilot study consisted of promoting multimodal handwashing and implementing strategies aimed at reducing HAI.

\section{Correspondencia:}

*Iván Renato Zúñiga-Carrasco

UMF 223

Av. Miguel Hidalgo

Col. La Mota

C.P. 52004, Lerma de Villada, Edo. de México, México

Date of reception: $28-05-2018$

E-mail: ivan.zuniga@imss.gob.mx

Date of acceptance: 12-09-2018

Doi: 10.24875/HMCM.18000130 
Prevalence of HAI was obtained from the monthly information issued by the hospital. A leader was assigned to coordinate the hand hygiene campaign through the Multimodal Strategy implemented by WHO. Results: From 2013 to 2017, the annual prevalence of HAl ranged from 60 to 119 cases. After the implementation of control strategies, nosocomial infections decreased. An important aspect was the correct classification of HAl. Conclusions: Pilot studies of this kind require 3 to 5 years to produce conclusive results. With the implemented strategies, adverse practices were changed, one of them being the over reporting of HAI. (HOSP MED CLIN MANAG. 2018;11:112-25)

Corresponding author: Iván Renato Zúñiga-Carrasco, ivan.zuniga@imss.gob.mx

Key words: Nosocomial infection. Strategy. Pilot test. Overregistration. Malpractice.

\section{INTRODUCCIÓN}

Las IAAS afectan en promedio al 10\% de los ingresos hospitalarios en todo el mundo, aunque se incrementan hasta el $25 \%$ en los países en vías de desarrollo, por lo que representan un problema relevante de salud pública a nivel internacional. En México hay un exceso de días en cama al año, estimado para el sector de la salud en 238,500 , lo cual representa un costo de $194,000,000$ pesos.

Durante 2013, en el Instituto Mexicano del Seguro Social (IMSS) se registraron 122,957 infecciones nosocomiales de 1,952,502 egresos hospitalarios, lo que representa 6.30 por cada 100 egresos. En los hospitales de segundo nivel se registró una tasa de infecciones de 5.8 por cada 100 egresos hospitalarios $(66,878 \mathrm{ca}-$ sos), 1.4 IN por caso. En las unidades médicas de alta especialidad (UMAE) se registró una tasa de 7.2 infecciones por 100 egresos (17,636 casos), 1.3 IN por caso. La letalidad asociada a las infecciones nosocomiales fue de 6.7 por $100 \mathrm{IN}$.

En segundo nivel, 6.7 por cada 100 infecciones (4,431 defunciones), y en las UMAE (1,221 defunciones) la letalidad fue de 6.9 por cada $100 \mathrm{IN}$, ocupando la cuarta causa de mortalidad en la población derechohabiente si se codificaran ${ }^{1}$.

También durante el año 2013, en el IMSS la tasa de neumonía con soporte ventilatorio mecánico en hospitales de segundo nivel fue de 18 a 29 casos por 1,000 días-ventilador, y en las UMAE fue de 10.07 casos por 1,000 días-ventilador ${ }^{1}$.

En el IMSS, la bacteriemia relacionada a catéter durante el año 2013 mostró una tasa global de 2.4 por 1,000 días-catéter; sin embargo, como ocurre en otros países en vías de desarrollo, el registro estadístico sigue siendo irregular ${ }^{1}$.

Durante 2012, en el IMSS la infección de sitio quirúrgico fue la infección nosocomial más frecuente (18.8\%), situación que se repitió durante el año 2013, en que fue del $20.6 \%{ }^{1}$.

En el IMSS, la infección de las vías urinarias asociada a sonda ocupa el tercer lugar de importancia entre las infecciones adquiridas intrahospitalariamente, con una tasa de 12 infecciones por cada 100 egresos ${ }^{1}$.

Datos de la OMS expresan que más de 1.4 millones de personas en el mundo contraen infecciones intrahospitalarias, entre el 5 y $10 \%$ de los pacientes que ingresan en los hospitales de países en desarrollo, donde el riesgo de adquirir una IAAS es de 2 a 20 veces mayor que en los países desarrollados. En México, se calcula que 450,000 casos de IAAS causan 32 muertes por cada 100,000 habitantes por año².

En países desarrollados, la prevalencia de pacientes hospitalizados que adquieren al menos una IAAS se encuentra entre un 3.5 y 12\%, mientras que en países en vía de desarrollo varía entre el 5.7 y $19.1 \%$, alcanzando en estos últimos una proporción incluso mayor al 25\% de pacientes afectados. En los servicios de unidades de cuidado intensivo $(\mathrm{UCl})$ de adultos, en países desarrollados se han documentado tasas acumuladas de infecciones relacionadas con el uso de ventilación mecánica, catéteres centrales y catéteres urinarios de 7.9, 3.5, 4.1 por 1,000 días-dispositivo ${ }^{3}$, respectivamente.

En 2011 se realizó un metaanálisis de la carga de enfermedad por las IAAS en los países desarrollados, demostrando que estos eventos representan un área de oportunidad para la seguridad del paciente. Se encontraron 
diferencias entre la prevalencia de IAAS entre EE.UU. (4.5\%), Canadá (11.6\%) y Europa (España, 8.1\%; Francia, 4.4\%; Suiza, 8.8\%; Reino Unido, 9\%; Bélgica, 6.9\%; Holanda, 7.2\%; Noruega, 5.1\%; Alemania, 3.6\%; Finlandia, 9.1\%; Eslovenia, 4.6\%; Italia, 6.7\%; Grecia, 7.9\%, y Nueva Zelanda, $12 \%)^{2,4}$.

En EE.UU., las principales IAAS son la infección de sitio quirúrgico, con un $20 \%$, la bacteriemia y la neumonía, ambas con un 11\%. En Europa, las IAAS que destacan principalmente son la infección de las vías respiratorias $(24 \%)$, seguida de la infección del sitio quirúrgico (17\%) y la bacteriemia (10.5\%). Según diversos estudios, la frecuencia de infección de sitio quirúrgico en otros países de altos ingresos se encuentra entre el 1.2 y el $5.2 \%{ }^{2}$

El European Center for Disease Prevention and Control, en un estudio realizado en hospitales de 17 países, reportó una prevalencia de IAAS del 3.4\%, la cual superó lo reportado en años anteriores. Asimismo, identificó que el $42.6 \%$ de estas instituciones contaban con un comité de control $^{5}$.

En Europa, datos del Programa de Seguimiento de Bacteriemias muestran que las IAAS afectan en promedio a 1 de cada 20 pacientes hospitalizados, es decir, 4.1 millones de pacientes, y de éstos se estima que unos 37,000 fallecen cada año por estas infecciones ${ }^{6}$.

En 2014 se encontró que, de un total de 110,945 pacientes, un 5.3\% adquirió neumonía en su estancia en la $\mathrm{UCl}$, el $92 \%$ de la cual estuvo asociada al uso de ventilador mecánico, por lo que la tasa de incidencia de la neumonía asociada al ventilador fue de 6.4 casos por 1,000 días-dispositivo. Pseudomona aureginosa, Staphylococcus aureus, Klebsiella pneumoniae, Escherichia coli y Candida spp fueron los microorganismos más aislados. Acinetobacter spp fue más frecuente en países como Italia, Portugal, Rumanía y Lituania 5 .

En cuanto a las infecciones del torrente sanguíneo en pacientes hospitalizados en $\mathrm{UCl}$, se encontró una tasa de incidencia de 3.3 casos por 1,000 días-paciente, de las cuales el $43.3 \%$ de los casos estuvieron relacionados con el uso del catéter venoso central. Los aislamientos de microorganismos más frecuentes fueron: Staphylococcus coagulasa negativos, Enterococcus spp, Staphylococcus aureus, Klebsiella spp, Pseudomona spp y Candida spp 5 .

El $3.1 \%$ de los pacientes que estuvieron hospitalizados en $\mathrm{UCl}$ presentaron infecciones del tracto urinario, y de éstas el 96.7\% estuvieron asociadas al uso de la sonda urinaria; la tasa se encontró en 3.9 casos por 1,000 días-dispositivo, el promedio del uso del catéter urinario fue del $83.9 \%$ y los microorganismos más frecuentes aislados fueron: Escherichia coli, Candida spp y Enterococcus $\mathrm{spp}^{5}$.

En América, datos de Canadá indican que se contraen unas 220,000 infecciones hospitalarias anuales, que dan lugar a 8,000 muertes relacionadas con esa causa ${ }^{7}$.

En EE.UU., las IAAS se encuentran entre las principales causas de muerte en el país, pues se estima que ocasionan 1.7 millones de infecciones y hasta 99,000 muertes al año $0^{3,8}$.

Datos del Center Desease Prevention and Control mostraron en $\mathrm{UCl}$ de adultos las tasas de incidencia de infecciones relacionadas con el uso de ventilación mecánica ( 1.1 casos), catéteres centrales (0.9 casos) y catéteres urinarios de (1.2 casos) por cada 1,000 días de uso $^{7}$.

En América Latina, a pesar de que las IAAS son una causa importante de morbilidad y mortalidad, países como Argentina ${ }^{9}$, Colombia $^{10}$, Brasil $^{11}$, Chile ${ }^{12}$ y Uruguay $^{13}$ tienen una vigilancia adecuada en sus servicios con datos instituciones y nacionales; sin embargo, sus objetivos, indicadores, definiciones y metodologías varían entre ellos.

En estudios puntuales realizados en algunos países de América se identificó que, entre las IAAS, la neumonía es la infección más frecuente, con una tasa de 24.1 casos por 1,000 días-ventilador, seguida por las infecciones asociadas a catéter venoso central (12.5 casos por 1,000 días-catéter) y las infecciones del tracto urinario asociadas con el uso de sondas vesicales (8.9 casos por 1,000 días-catéter urinario) ${ }^{14}$.

Adicional al impacto que las IAAS causan en la calidad de vida de los pacientes, se tiene la carga económica atribuible a las mismas ${ }^{15}$. 
Se estima que al día 1.5 millones de personas en el mundo padecen complicaciones infecciosas relacionadas con la atención hospitalaria. Cada día 247 personas mueren en EE.UU. por una infección adquirida durante su atención médica.

Tres cuartas partes de las infecciones nosocomiales son altamente prevenibles con el lavado de manos. La OMS ha establecido directrices sobre la higiene de las manos en la atención sanitaria, y partiendo de estos datos, existen una serie de componentes que constituyen una estrategia multimodal eficaz para la higiene de las manos. Se ha propuesto que la estrategia multimodal para la mejora de la higiene de las manos lleve a la práctica las recomendaciones, por lo que esta estrategia va acompañada de una amplia serie de herramientas prácticas listas para ser usadas para su aplicación.

Los componentes de la estrategia son:

- Cambio del sistema: garantizar que se cuenta con la infraestructura necesaria para permitir a los profesionales sanitarios practicar la higiene de las manos. Esto incluye dos elementos esenciales:

- Acceso a un suministro seguro y continuo de agua, así como a jabón y toallas.

- Fácil acceso a la solución a base de alcohol (SBA) para manos en el punto de atención.

- Formación: proporcionar formación con regularidad a todos los profesionales sanitarios sobre la importancia de la higiene de las manos, basada en el modelo de «los cinco momentos para la higiene de las manos», y los procedimientos adecuados para la fricción y/o lavado de manos.

- Evaluación y retroalimentación: hacer un seguimiento de las infraestructuras y prácticas de higiene de manos, junto con las correspondientes visiones y conocimientos por parte de los profesionales de la salud, y al mismo tiempo proporcionar a todo el personal de salud información sobre los resultados del impacto del programa.

- Recordatorios en el lugar de trabajo: señalar y recordar a los profesionales de la salud la importancia de la higiene de las manos y las indicaciones y procedimientos adecuados para llevarla a cabo.
- Clima institucional de seguridad: crear un entorno y percepciones que propicien la sensibilización sobre las cuestiones de seguridad del paciente, y garantizar al mismo tiempo que la mejora de la higiene de las manos se considere una gran prioridad a todos los niveles. Esto supone:

- Participación activa a escala institucional e individual.

- Toma de conciencia de la capacidad individual e institucional de cambiar y mejorar (autoeficacia).

- Asociación con pacientes y organizaciones de pacientes $^{16}$.

\section{Metodología}

Se realizó una prueba piloto en el Hospital General Regional \# 251 que consistió en impulsar el lavado de manos multimodal e implementar estrategias encaminadas a reducir las IAAS. Los resultados de dicha prueba piloto se midieron a través de la información mensual que emite el hospital sobre las IAAS, la cual se recopila de los estudios de caso (Figs. 1 y 2) que las enfermeras especialistas en salud pública (EESP) captan en su recorrido diario en los diferentes servicios hospitalarios, tanto en búsqueda activa como en búsqueda intencionada. Se asignó un líder, encargado de coordinar la campaña de higiene de mano a través de la estrategia multimodal implementada por la OMS.

\section{ESTRATEGIAS IMPLEMENTADAS EN LA PRUEBA PILOTO}

\section{Higiene de manos}

- Se capacitó al 100\% del personal de la unidad en relación con la higiene de las manos.

- Se estableció una base de datos que identificó de manera confiable el personal que fue capacitado y que realizó todos los cuestionarios.

- A todos los participantes se les otorgó un frasco unidosis con gel desinfectante con SBA para que lo portaran en toda su jornada laboral; una vez terminada esta, lo canjearían por uno nuevo (Fig. 3). 


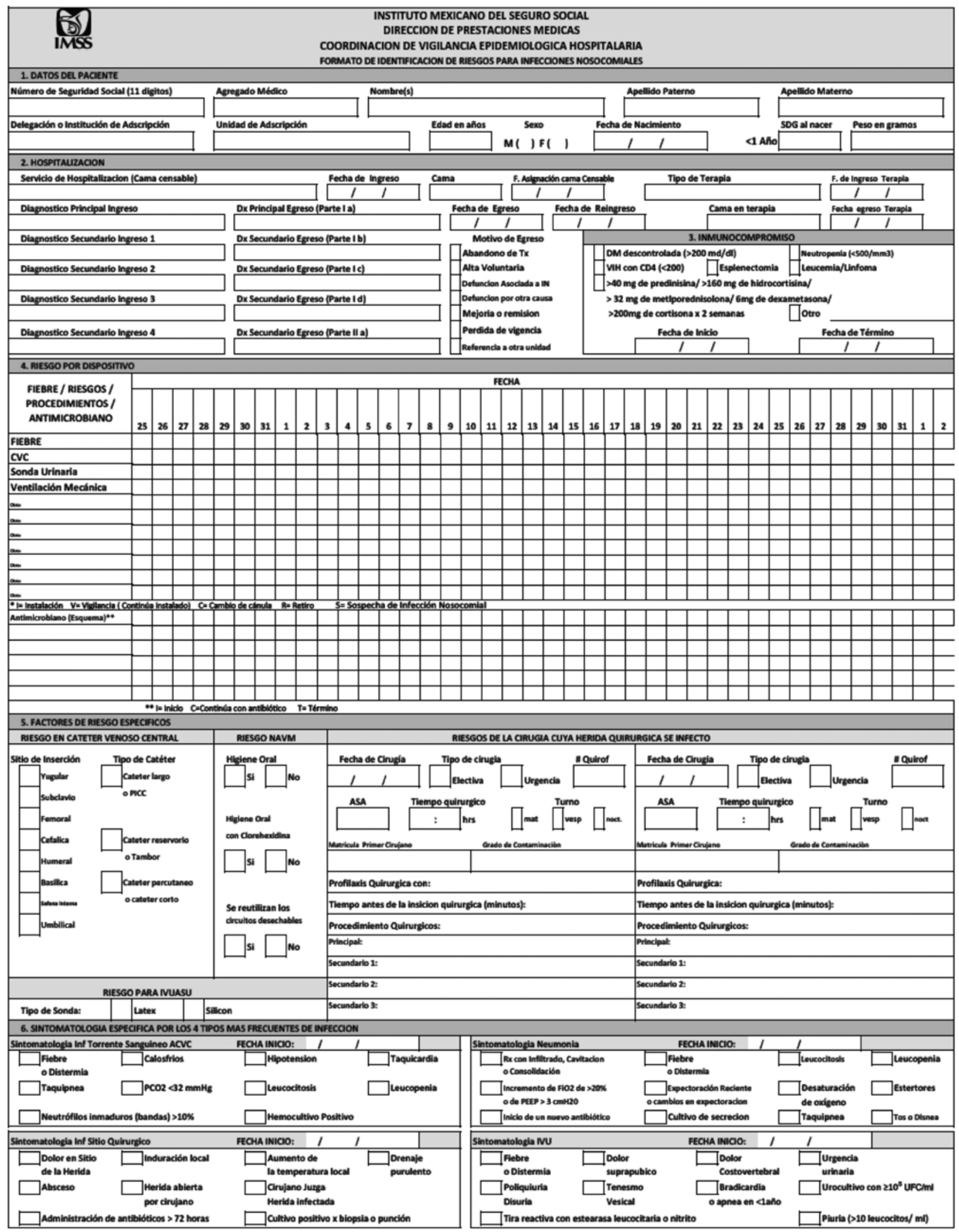

EUBoro:

Figura 1. Registro de caso de infección nosocomial. Cara anterior. 


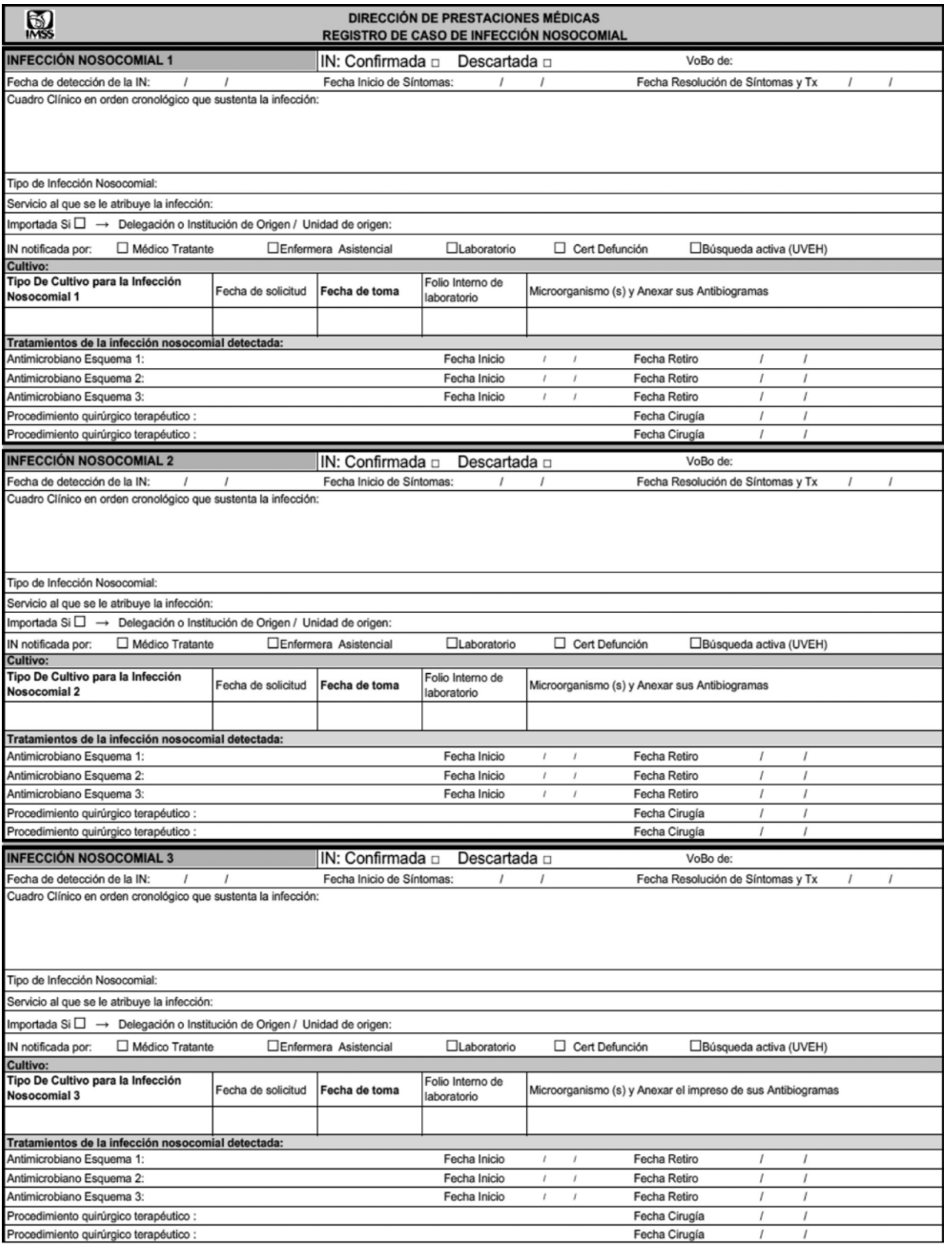

Figura 2. Registro de caso de infección nosocomial. Cara posterior. 


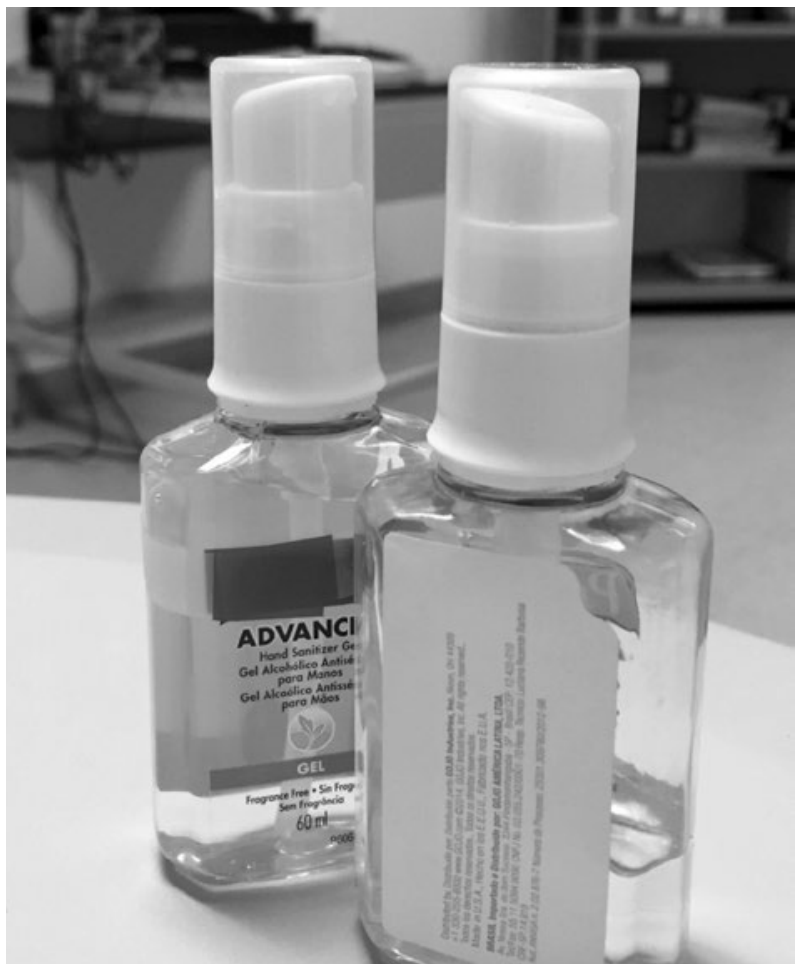

Figura 3. Frasco unidosis con gel desinfectante.

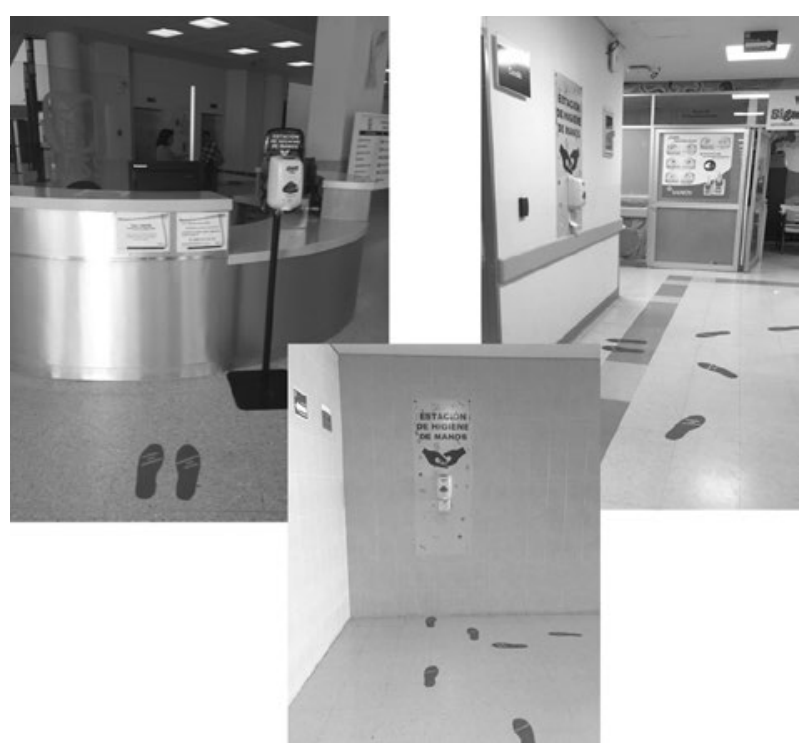

Figura 4. Siluetas de pisadas autoadheribles en los puntos de mayor tránsito.

- En la UCl y Área de Choque la solución debía ser a base de clorhexidina.

- Se colocaron carteles alusivos sobre: «Lavado de manos con agua y jabón», "Los cinco momentos», «Fricción de manos con solución a base de alcohol» en los puntos de mayor afluencia y de fácil acceso tanto de personal que labora en el hospital como de

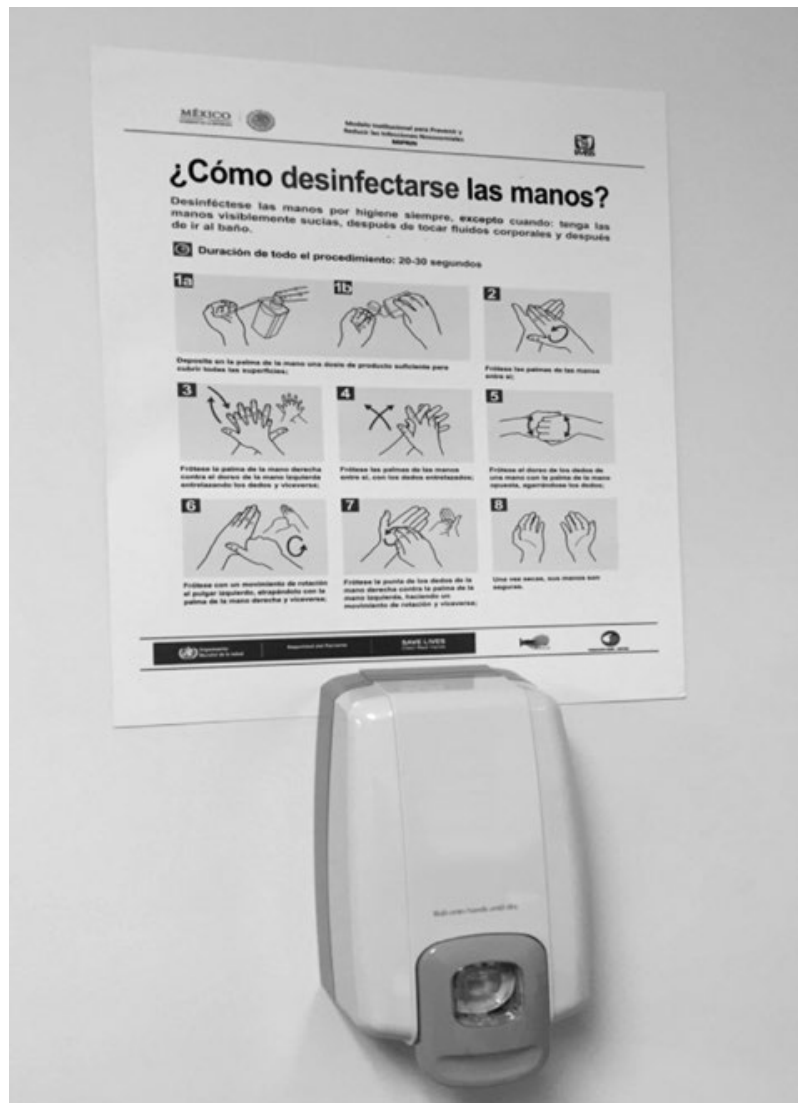

Figura 5. Dispensadores de solución a base de alcohol con cartel de técnica de higiene de manos.

visitantes. Para que el impacto visual fuera exitoso en estos puntos, se elaboraron siluetas de pisadas autoadheribles para ser colocadas en el suelo, principalmente en los puntos de mayor tránsito, con el fin de que dicho señalamiento dirigiera a las personas al dispensador de SBA (Figs. 4 y 5).

- Se colocaron dispensadores de SBA, de igual manera que los carteles, cerca de los lavamanos, en cada cubículo, al lado de la cama del paciente (Fig. 6).

- Se incrementó la compra de lavamanos, dispensadores para jabón líquido de manos y dispensadores para toallas de papel desechables para colocarlos en varios puntos del hospital.

- Periódicamente se supervisaba que hubiese abasto suficiente de insumos, funcionalidad de lavamanos y dispensadores.

- Semanalmente se pesaban las SBA y el jabón con el fin detectar el consumo de los mismos. 


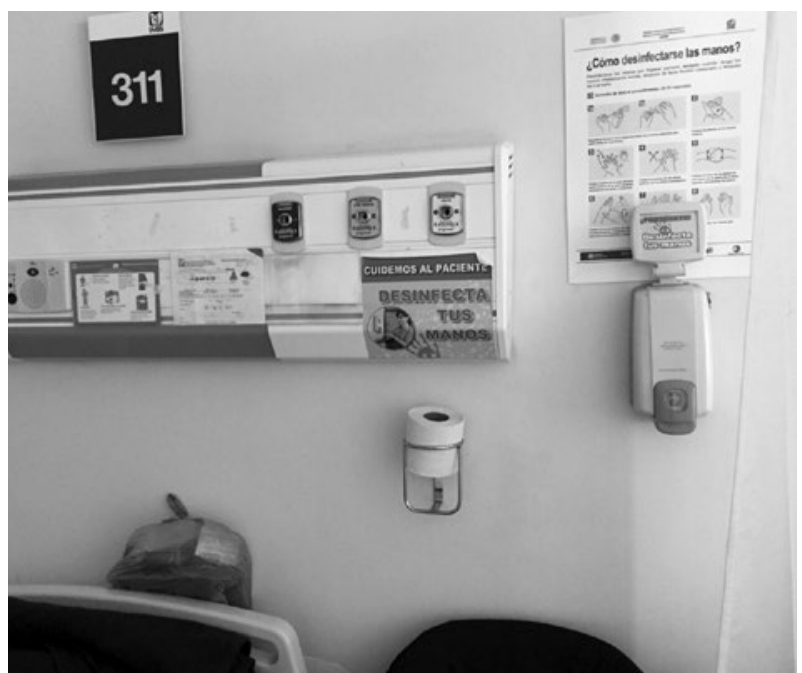

Figura 6. Cubículos con dispensador y cartel de higiene de manos.

- Se colocó un dispensador de crema hipoalergénica en el área de checadores para aquellas personas que presentaran resequedad en las manos.

\section{Detección de infecciones asociadas a la atención a la salud}

- El número de EESP en la Unidad de Vigilancia Epidemiológica Hospitalaria (UVEH) era permanente: cuatro en turno matutino y una en turno vespertino. En caso de que alguna de ella estuviera ausente, se asignaba una enfermera para no dejar descubierta la plaza. En el turno especial, fines de semana y festivos, había una enfermera asignada a la UVEH.

- Cada servicio colocó un pizarrón de plumón, en el cual a diario se apuntaban los probables casos de IAAS detectados, tanto por enfermería como por el médico tratante, validados por los jefes de servicio (Fig. 7).

- Se asignaron dos capturistas con su equipo propio de cómputo para la captura de la información, a los que se les entregaban diariamente hojas de pacientes hospitalizados como hoja de estudio de caso de IAAS.

- Como sustento bibliográfico para la adecuada detección de IAAS, se tomaron en cuenta el «Manual de Criterios para el diagnóstico de las Infecciones Asociadas a la Atención de la Salud (IAAS)», Norma Oficial Mexicana NOM-045-SSA2-2005, Para la

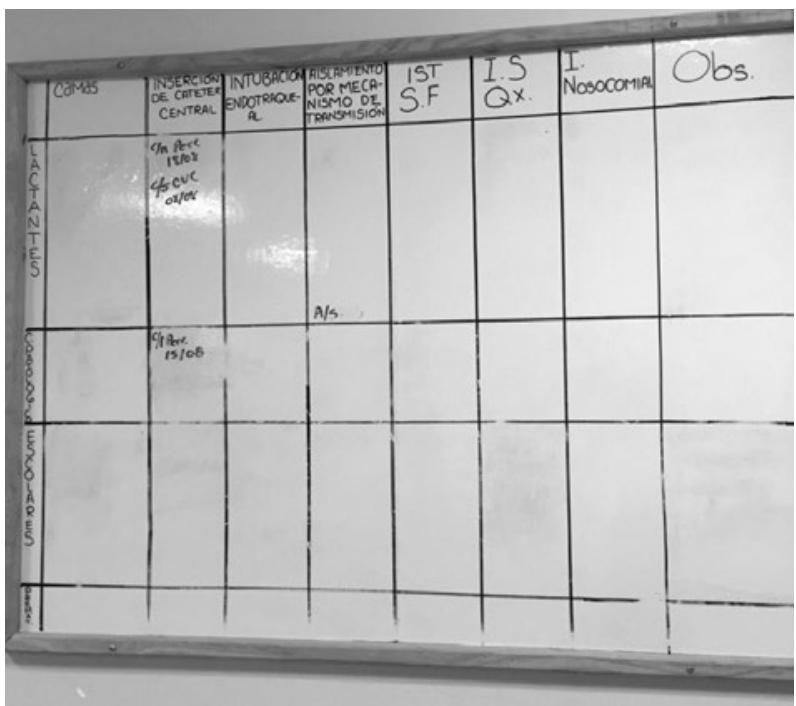

Figura 7. Pizarrón para notificar diariamente IAAS.

vigilancia epidemiológica, prevención y control de las infecciones nosocomiales, Guidelines for the Prevention of Intravascular Catheter-Related Infections 2011, Guideline for prevention of catheterassociated Urinary tract infections 2009, Strategies to Prevent Surgical Site Infections in Acute Care Hospitals: 2014 Update y Guidelines for the Management of Adults with Hospital-acquired, Ventilator-associated y Healthcare-associated Pneumonia.

- La hoja de estudio de caso de IAAS cuenta con los criterios esenciales del documento antes comentado con el fin de evitar subregistros y sobrerregistros.

\section{Procesamiento de la información}

- Se estableció una base de datos para la captura de todos los pacientes hospitalizados tomando en cuenta las cuatro infecciones de gran impacto estableciendo variables clave:

- Infección de sitio quirúrgico: fecha de cirugía, tiempo quirúrgico, clasificación de la American Society of Anesthesiologists, clasificación del tipo de herida quirúrgica, uso de antibióticos profilácticos, tricotomía del sitio quirúrgico, procedimiento quirúrgico.

- Neumonía asociada a ventilador y no asociada a ventilación mecánica: fecha de instalación, fecha de retiro, fecha de reinstalación, fecha de retiro definitivo, días-dispositivo, higiene oral, elevación 
de la cabecera, sedación [fecha de inicio, fecha de retiro, días de sedación], días totales de ventilación mecánica.

- Infección del sitio del catéter: catéter venoso central, fecha de inserción, fecha de retiro, días-dispositivo, sitio de inserción, numero de lúmenes, datos de la infección en el sitio de inserción, días totales de catéter venoso central.

- Infección de vías urinarias asociada a sonda vesical y no asociada: fecha de instalación, fecha de retiro, fecha de reinstalación, fecha de retiro definitivo, días-dispositivo, motivo de instalación de la sonda vesical, se mantiene el sistema cerrado, total días sonda vesical.

- Las información mensual que generaba el hospital de todos los servicios tenía que ser emitida oportunamente para tener el informe mensual de la prueba piloto. Dicha información constaba de: número de egresos hospitalarios, número total de defunciones, total de días de estancia hospitalaria (se desglosaba en los cuatro servicios troncales: Cirugía, Medicina Interna, Pediatría, Ginecoobstetricia) -sin omitir los días de estancia hospitalaria y egresos en la Unidad de Terapia Intensiva, que incluye adultos, pediatría y neonatos-, número de cirugías y número de cirugías limpias.

\section{RESULTADOS}

Los reportes mensuales hospitalarios de IAAS del año 2013 se tomaron como comparativos para la prueba piloto que se realizó en 2016; el número de casos de IAAS fluctuaba entre los 60 y 119 casos. Al implementar las estrategias para la disminución de las infecciones nosocomiales, siendo una de ellas su correcta clasificación, disminuyeron los casos. El año 2017 se dejó como año de fortalecimiento de todas las estrategias tomadas de la prueba piloto, y se puede observar que continúa la disminución después de la prueba y que ésta es mayor en comparación con los años previos (Fig. 8)

Con respecto a la neumonía asociada a la ventilación mecánica, infección del sitio del catéter e infección de las vías urinarias por sonda vesical, existió disminución mientras se realizaba la prueba piloto, pero en al año
2017 nuevamente se incrementó el número, ya que las infecciones con medios invasivos son las que más impacto tienen con respecto a las IAAS (Figs. 9-11).

Con respecto a la bacteriemia, continúo disminuyendo el número de casos, comparando la prueba piloto y años previos (Fig. 12).

Con respecto a la infección del sitio quirúrgico, se mantuvo el mismo número de casos posterior a la prueba piloto, disminuyendo con respecto al año 2015 (Fig. 13).

Con respecto a la $\mathrm{UCl}$, los casos disminuyeron en comparación a los años previos a la prueba piloto (Fig. 14).

Con respecto a la higiene de las manos, se obtuvieron los siguientes datos:

- Durante la prueba piloto mensualmente se realizaba una reunión para analizar la información, corregir y tomar nuevas estrategias. Podemos observar que en el mes de mayo hay un descenso de un 3.3\% con relación al mes de abril; aquí las estrategias que se tomaron fueron:

- Las enfermeras sanitaristas, en el momento de detectar una IAAS, se basaron en los criterios clínicos y diagnósticos que vienen en el estudio del caso, ya que anteriormente se utilizaba uno en el cual se pautaba para ingresar cualquier infección nosocomial aunque no cumpliera los criterios contenidos en el «Breviario para la Vigilancia Epidemiológica de las Infecciones Asociadas a la Atención de la Salud, su Prevención y Control», ya que en la búsqueda activa e intencionada de IAAS, aunque el médico tratante la notificara tanto en el expediente como en la interconsulta, se captaba sin analizarla. Cada mes se reunían los jefes de servicio, la directora, el líder de la Campaña Institucional de Higiene de Manos y el epidemiólogo, junto con los líderes del MIPRIN, para validar y analizar, según los criterios del breviario, las infecciones captadas por las EESP con el expediente del paciente. Al final se validaban las infecciones que correspondían a cada servicio sin descartar arbitrariamente infecciones con tal de tener menos. Se pudo detectar que una enfermera que suplía a las EESP cuando se ausentaban o estaban de vacaciones «detectaba» infecciones, pero en el momento de la validación se encontró que muchas de ellas eran 


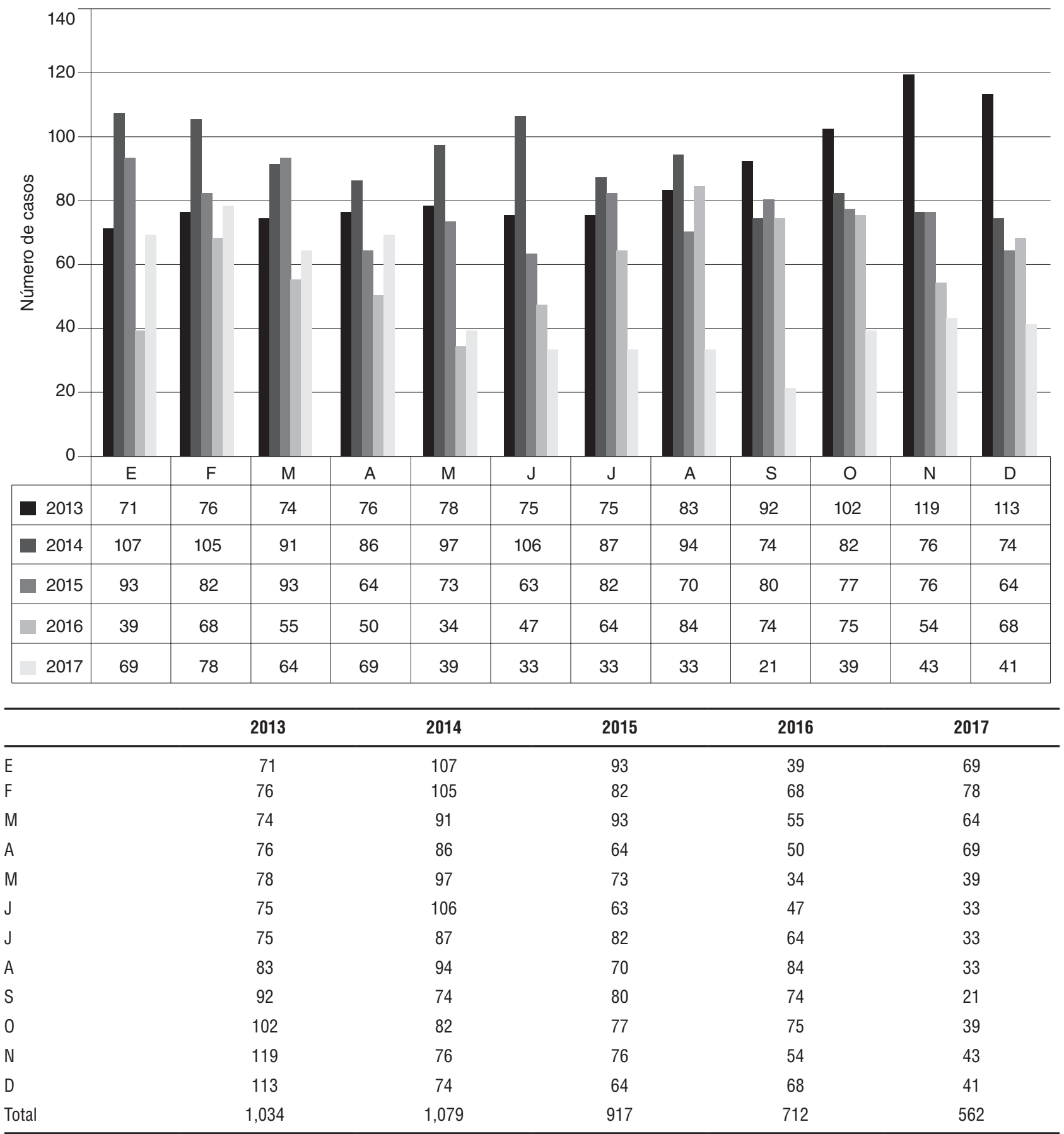

Figura 8. Reportes mensuales hospitalarios de IAAS 2013-2017.

falsas e improvisadas, las cuales se descartaban cuando se leían las notas del expediente. A dicha enfermera se le dio de baja como personal de ayuda al Servicio de Epidemiología, ya que su información ponía en riesgo no sólo la prueba piloto, sino también posteriormente si se le incluía en la UVEH ${ }^{17}$.

\section{CONCLUSIONES}

Las pruebas piloto requieren unos 3-5 años para ver resultados concluyentes. Con las estrategias implementadas se cambiaron malas prácticas, una de ellas fue el sobrerregistro de IAAS, ya que al no estar validadas tanto por el jefe de servicio como por el epidemiólogo, 


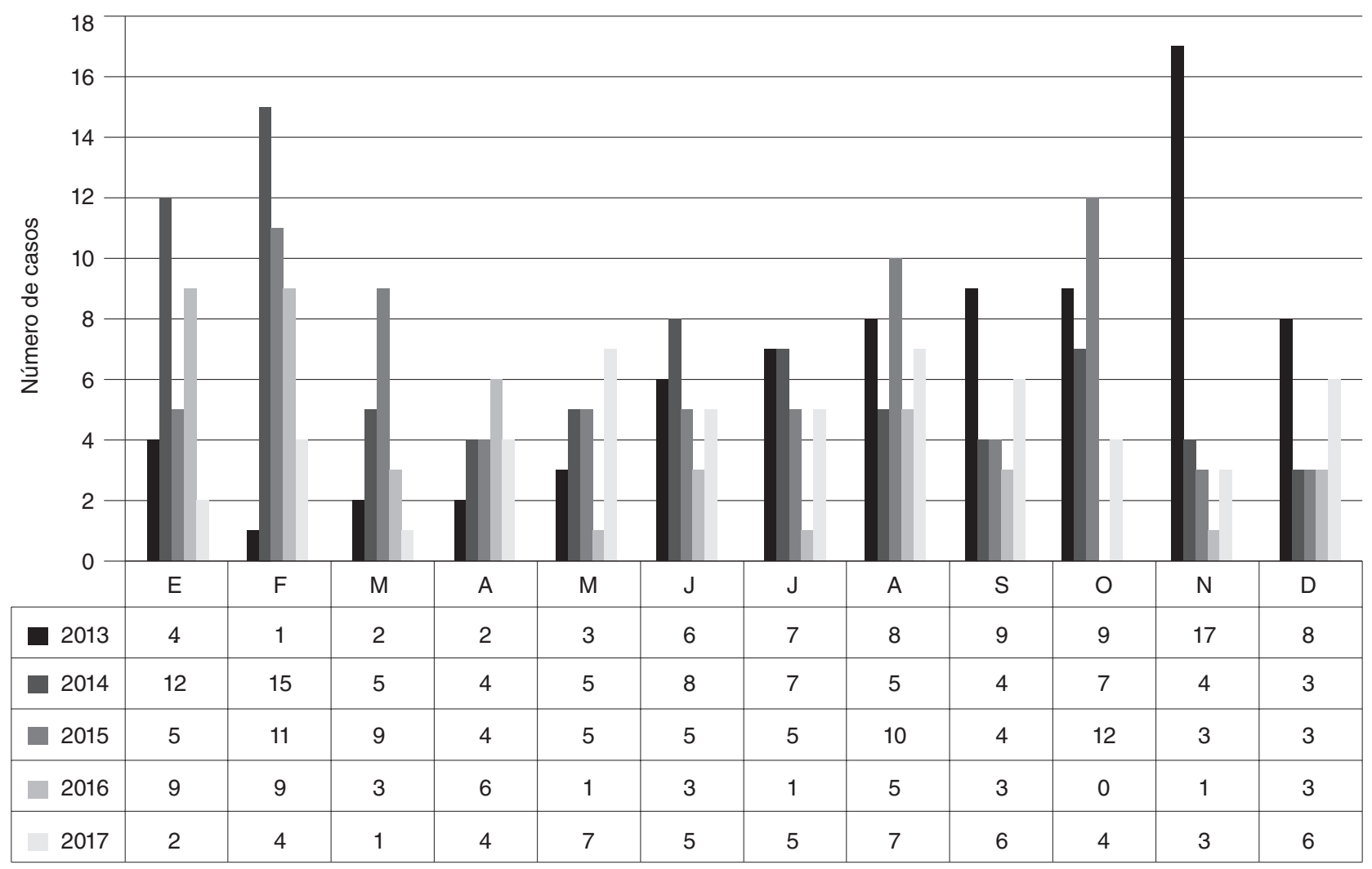

Figura 9. NAVM. Reporte mensual de neumonía asociada a la ventilación mecánica (NAVM) 2013-2017.

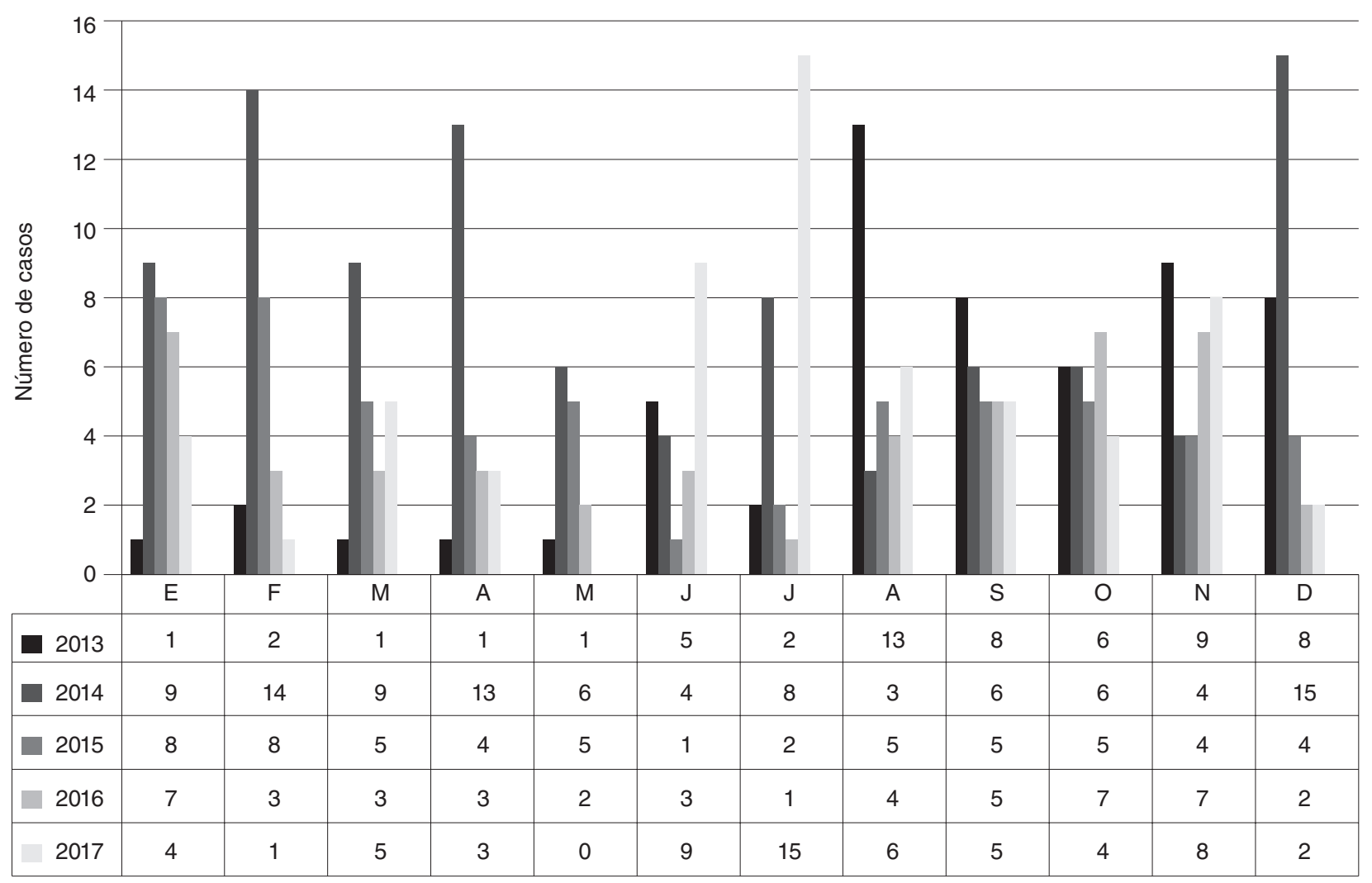

Figura 10. Reporte mensual de infección del sitio de catéter 2013-2017. 


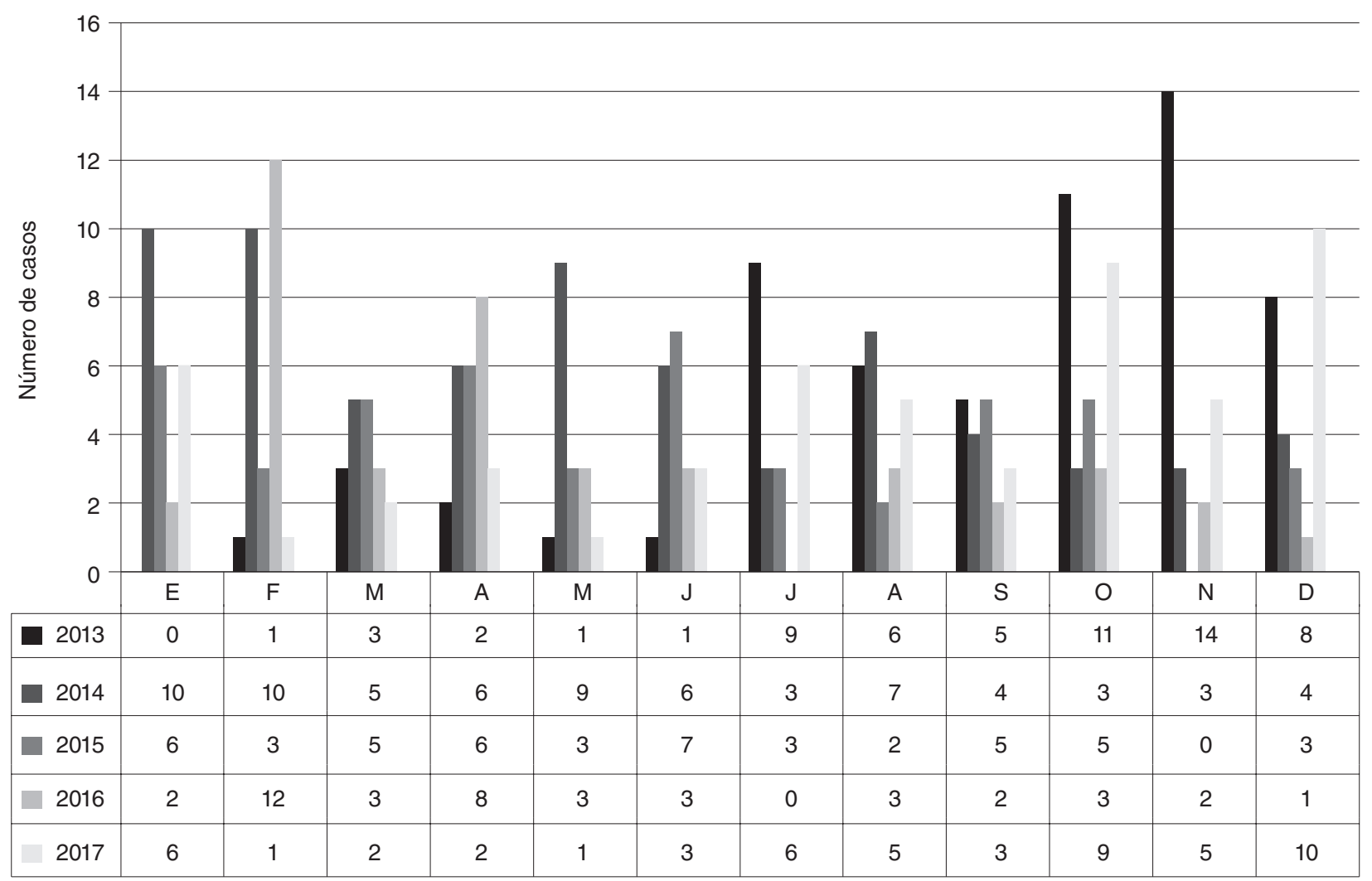

Figura 11. Reporte mensual de infección de vías urinarias por sonda vesical (IVUSV) 2013-2017.

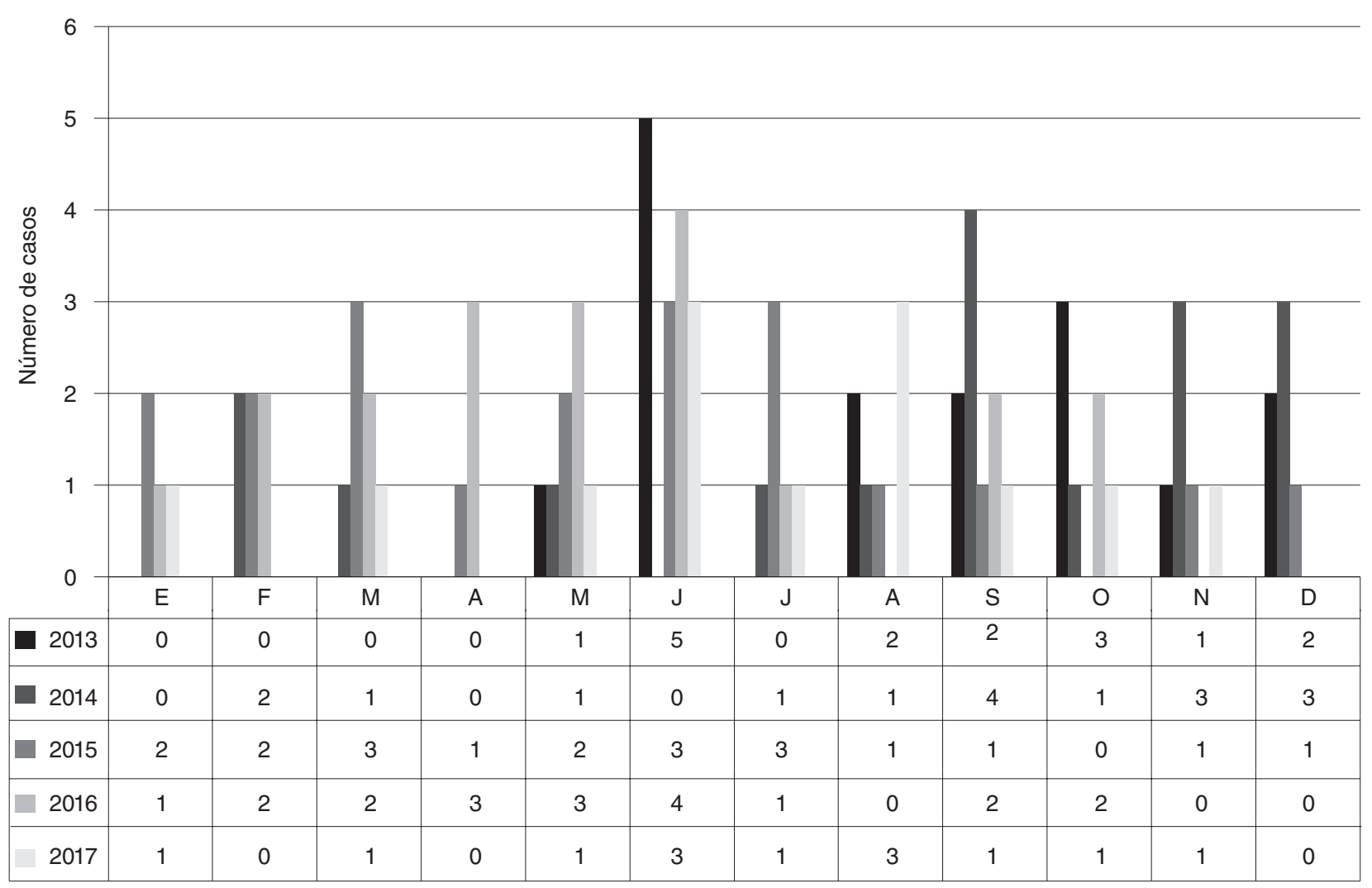

Figura 12. Reporte mensual de bacteremia 2013-2017. 


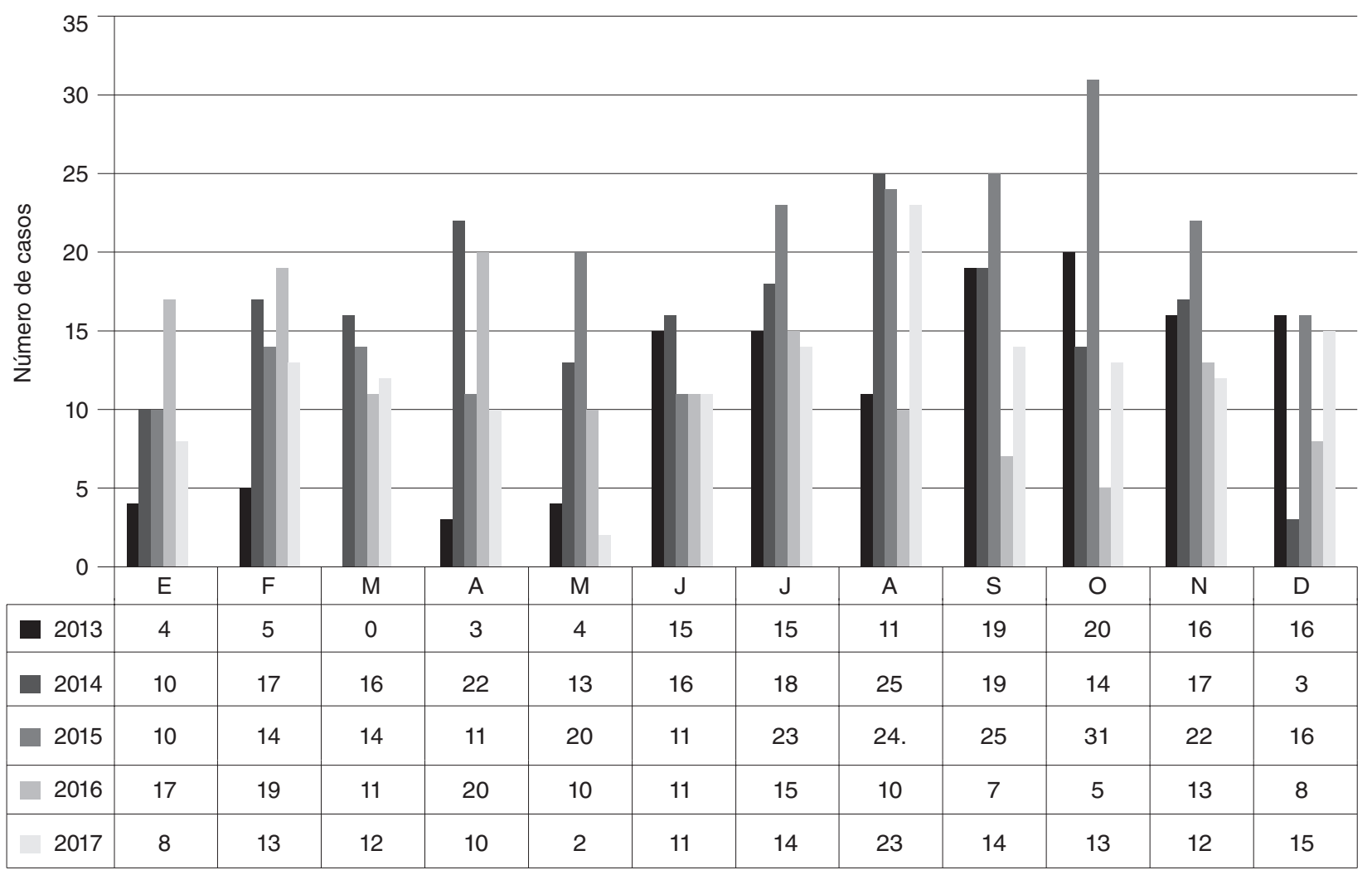

Figura 13. Reporte mensual de heridas de sitio quirúrgico 2013-2017.

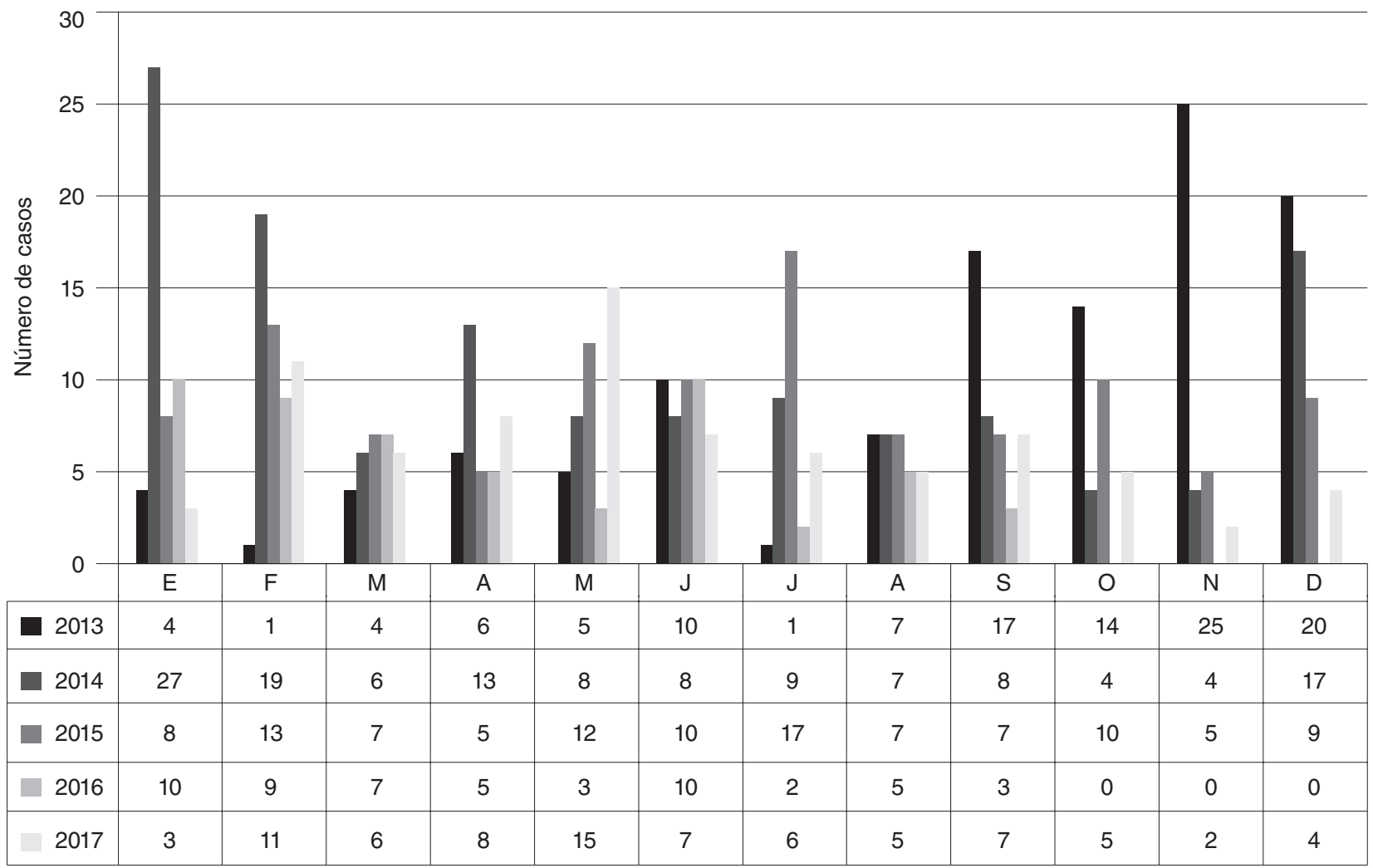

Figura 14. Reporte mensual de IAAS en Unidad de Cuidados Intensivos (UCI) 2013-2017. 
los registros mensuales siempre estarían en incremento. Así pues, una adecuada hoja del caso es importante para poder registrar adecuadamente el tipo de infección nosocomial para darle seguimiento diario hasta la resolución o egreso del paciente, y tampoco hay que olvidar las acciones encaminadas para prevenir las IAAS, que incluyen: adecuadas técnicas de limpieza y desinfección, las cuales deben ser en los tres turnos, así como los fines de semana, siempre teniendo un óptimo manejo de soluciones desinfectantes; que un equipo multidisciplinario realice recorridos cada tres días para poder detectar áreas de oportunidad y factores de riesgo que puedan incrementar las IAAS y desencadenar un brote nosocomial; personal suficiente, principalmente de enfermería, con el fin de evitar infecciones cruzadas; dotación suficiente de insumos para la higiene de manos; calidad del agua intrahospitalaria adecuada para todo lo que ella implica en su uso -importancia del monitoreo de la cloración y bacteriológico, realizar cultivos de superficie y ambientales para la detección oportuna de patógenos y realizar estudios de tolerabilidad-; capacitación de personal de nuevo ingreso, becarios y estudiantes en el tema de la higiene de mano e IAAS, y concienciar a todo el personal operativo que labora dentro del nosocomio, así como a familiares de pacientes y personal que se encuentre visitando dicho edificio, para llevar a cabo «Los cinco momentos», punto fundamental para poder disminuir las IAAS.

\section{AGRADECIMIENTOS}

A los jefes de los servicios del Hospital General Regional \# 251, al equipo de UVEH (Teresa de Jesús Díaz Villegas, Violeta López Sánchez, Blasa Silvia Martínez Romero, Marisol Elena Díaz García y Brenda Idalia González Posadas) y a los capturistas (Gabriela Mercado Valdez, Adrián Zepeda Bernal y Jonathan Pérez Gutiérrez).

Los autores declaran que dicha prueba no tuvo ningún financiamiento.

\section{BIBLIOGRAFÍA}

1. Cote E. MIPRIN Planteamiento General. Simposio Internacional de Higiene de mano; 2014 agosto; México.

2. Organización Panamericana de la Salud. Una atención más limpia es una atención más segura. [Consultado el 2 de mayo de 2018]. Disponible en: http://www.who.int/gpsc/background/es/

3. World Health Organization. Report on the Burden of Endemic Health Care-Associated Infection Worldwide 2011. [Consultado el 2 de mayo de 2018]. Disponible en: http://apps.who.int/iris/bitstream/10665/80135/1/9789241501507 eng.pdf

4. Allegranzi B, Bagheri N, Combescure C, Graafmans W, Attar H, Donaldson $\mathrm{L}$, et al. Burden of endemic health-care-associated infections in developing countries: systemic review and meta-analisys. Lancet. 2011;377(9761): 228-41.

5. ECDC. Annual epidemiological report 2014- Antimicrobial resistance and healthcare-associated infections. [Consultado el 2 de mayo de 2017]. Disponible en: http://ecdc.europa.eu/en/publications/Publications/antimicrobial-resistance-annual-epidemiological-report.pdf

6. Organización Panamericana de la Salud. Vigilancia de las Infecciones Asociadas a la Atención en Salud, Módulo III: Información para gerentes y directivos. [Consultado el 2 de mayo de 2017]. Disponible en: http://www2. paho.org/hq/index.php?option=com_docman\&task=doc_view\&gid=22315 \&itemid\&ltemid $=270$

7. Dudeck M, Weiner L, Allen K, Malpiedi P, Peterson K, Pollock D, et al. National Healthcare Safety Network (NHSN) report, data summary for 2012, Device-associated module. Am J Infect Control. 2013:41(12):1148-66.

8. Klevens R, Edwards J, Richards C, Horan T, Gaynes R, Pollock D, et al. Estimating healthcare-associated infections in U.S. hospitals, 2002. Public Health Rep. 2007:122(2):160-6.

9. Ministerio de Salud de la Nación de Argentina. Programa Nacional de Epidemiología y Control de Infecciones Hospitalarias de Argentina. [Consultado el 2 de mayo de 2018]. Disponible en: http://www.msal.gob.ar/ images/stories/cofesa/2008/acta-02-08/anexo-10-acta-02-08.pdf

10. Detectar, prevenir y reducir infecciones asociadas con la atención en salud. [Consultado el 2 de mayo de 2018]. Disponible en: https://www.minsalud. gov.co/sites/rid/Lists/BibliotecaDigital/RIDE/DE/CA/Detectar-Infecciones.pdf

11. Programa nacional de Prevenção e controle de Infecções relacionadas à Assistência à saúde. [Consultado el 2 de mayo de 2018]. Disponible en: http://portal.anvisa.gov.br/documents/33852/272166/Programa+Nacionalde+Preven $\%$ C3\%A7\%C3\%A30+e+Controle+de+Infec $\%$ C3\%A7\%C3\%B5es+ Relacionadas+ $\%$ C3\%A0+Assist $\%$ C3 $\%$ AAncia $+\%$ C3 $\%$ A0 + Sa $\%$ C $3 \%$ BA de+\%282013-2015\%29/d1d0601f-004c-40e7-aaa5-0af7b32ac22a

12. Ministerio de Salud de Chile. Infecciones Intrahospitalarias. [Consultado el 2 de mayo de 2017]. Disponible en: http://web.minsal.cl/infecciones_intrahospitalarias/

13. Ministerio de Salud Pública de Uruguay. Control de infecciones intrahospitalarias. [Consultado el 2 de mayo de 2018]. Disponible en: http://www. msp.gub.uy/publicaciones/direcci\%C3\%B3n-general-de-salud/ divisi\%C3\%B3n-epidemiolog\%C3\%ADa/control-infecciones

14. Rosenthal VD, Maki DG, Salomao R, Moreno CA, Mehta Y, Higuera F, et al. Device-associated nosocomial infections in 55 intensive care units of 8 developing countries. Ann Intern Med. 2006;145(8):582-91.

15. U.S. Department of Health and Human Services. National Action Plan to Prevent Healthcare-associated infections: Road Map to Elimination. [Consultado el 2 de mayo de 2017]. Disponible en: https://health.gov/hcq/prevent-hai-action-plan.asp

16. Organización Mundial de la Salud. Guide to Implementation. A Guide to the Implementation of the WHO Multimodal Hand Hygiene Improvement Strategy. 2009 [Consultado el 2 de mayo de 2018]. Disponible en: http://www. who.int/gpsc/5may/Guide to Implementation.pdf

17. Breviario par la Vigilancia Epidemiológica de las Infecciones Asociadas a la Atención de la Salud, su Prevención y Control. Dirección de Prestaciones Médicas. Unidad de Salud Pública. Coordinación de Vigilancia Epidemiológica. Instituto Mexicano del Seguro Social. 1. ${ }^{a}$ ed. 2017. 